\title{
Optimally Efficient Bidirectional Search
}

\author{
Eshed Shaham $^{1 *}$, Ariel Felner ${ }^{2}$, Nathan R. Sturtevant ${ }^{3}$ and Jeffrey S. Rosenschein ${ }^{1}$ \\ ${ }^{1}$ School of Engineering and CS, Hebrew University of Jerusalem, Jerusalem, Israel \\ ${ }^{2}$ SISE Department, Ben-Gurion University of the Negev, Be'er Sheva, Israel \\ ${ }^{3}$ CS Department, University of Alberta, Canada \\ eshed.shaham@mail.huji.ac.il,felner@bgu.ac.il,nathanst@ualberta.ca,jeff@cs.huji.ac.il
}

\begin{abstract}
$\mathrm{A}^{*}$ is optimally efficient with regard to node expansions among unidirectional admissible algorithms - those that only assume that the heuristic used is admissible. This paper studies algorithms that are optimally efficient for bidirectional search algorithms. We present the Fractional MM algorithm and its sibling, the MT algorithm, which is simpler to analyze. We then develop variants of these algorithms that are optimally efficient, each under different assumptions on the information available to the algorithm.
\end{abstract}

\section{Introduction and Background}

Given a graph $G$, the shortest-path problem is to find the leastcost path from state start to state goal in $G$. The traditional approach is to use the A* algorithm [Hart et al., 1968]. A* is a unidirectional best-first search that priorities nodes according to $f(n)=g(n)+h(n)$ where $g(n)$ is the shortest known path from start to the node $n$ and $h(n)$ is an admissible heuristic (lower bound) on the cost of the path from $n$ to the goal.

Bidirectional heuristic search algorithms (denoted henceforth by $\mathrm{Bi}-\mathrm{HS}$ ) interleave two separate searches, a search forward from $s$ and a search backward from $g$. these algorithms usually halt once both frontiers include the same node. Bi-HS algorithms differ on which node they expand next and when exactly they stop such that optimality is guaranteed (see a survey in [Holte et al., 2017]).

Recent research defined conditions on the node expansions required by $\mathrm{Bi}-\mathrm{HS}$ algorithms to guarantee solutions optimality [Eckerle et al., 2017]. Following work reformulated these conditions as a must-expand graph $\left(G_{\mathrm{MX}}\right)$, showing that the Minimum Vertex Cover (MVC) of $G_{\mathrm{MX}}$ corresponds to the minimal number of expansions required to prove optimality [Chen et al., 2017]. In this paper we study the $G_{\mathrm{MX}}$ struc-

${ }^{*}$ This paper is a is a summary of two papers: "The Minimal Set of States that Must Be Expanded in a Front-to-End Bidirectional Search" [Shaham et al. 2017] and "Minimizing Node Expansions in Bidirectional Search with Consistent Heuristics" [Shaham et al. 2018]. Both paper were presented at the Symposium of Combinatorial Search (SoCS). These papers are are the basis for the M.Sc thesis of the first author. ture, characterize properties of the MVC and develop algorithms that expand exactly the MVC.

Let $d(x, y)$ denote the shortest distance between $x$ and $y$ and let $d($ start, goal $)=C^{*}$.

We use $f_{F}, g_{F}$ and $h_{F}$ to indicate $f-, g$-, and $h$-values in the forward direction and similarly, $f_{B}, g_{B}$ and $h_{B}$ to indicate $f-, g$-, and $h$-values in the backward direction. The forward heuristic, $h_{F}$, is forward admissible iff $h_{F}(u) \leq d(u$, goal $)$ for all $u$ in $G$ and is forward consistent iff $h_{F}(u) \leq d\left(u, u^{\prime}\right)+$ $h_{F}\left(u^{\prime}\right)$ for all $u$ and $u^{\prime}$ in $G$. The backward heuristic, $h_{B}$, is backward admissible iff $h_{B}(v) \leq d($ start, $v)$ for all $v$ in $G$ and is backward consistent iff $\bar{h}_{B}(v) \leq d\left(v^{\prime}, v\right)+h_{B}\left(v^{\prime}\right)$ for all $v$ and $v^{\prime}$ in $G$. Front-to-end algorithms use these two heuristic functions coupled with the $g$-value of the corresponding node ( $v$ or $u$ ). Front-to-front bidirectional search algorithms use heuristics between pairs of states on opposite frontiers coupled with their respective $g$-values.

Let $I_{A D}$ be the set of solvable problem instances in which the heuristics are admissible, and let $I_{C O N}$ be the subset of problems in $I_{A D}$ where the heuristics are consistent. Admissible algorithms are algorithms that must be able to optimally solve all instances from $I_{A D}$. Admissible algorithms have no further knowledge about the problem or the heuristic (besides the fact that it is admissible), and they do not exploit such knowledge even if it is available. We deal with three different settings distinguished by the assumptions on the algorithms and the problem instances.

Case 1: Recent papers on bidirectional search (e.g., [Eckerle et al., 2017]), followed the classic analysis of $\mathrm{A}^{*}$ [Dechter and Pearl, 1985] and analyzed admissible algorithms running on problem instances with consistent heuristics. That is, the behavior of admissible algorithms was only studied when running on instances from $I_{C O N}$. We denote these assumptions as the base case and label it by $I_{A D} / I_{C O N}$ along the following notation: What can be assumed by the algorithm on the problem instances / The instances the algorithm is executed on.

Case 2: In many domains (e.g., with unit-cost edges) the cost of the smallest edge $(\epsilon)$ is known. We label this case, where the algorithm knows $\epsilon$ and is allowed to exploit this knowledge as $I_{A D \epsilon} / I_{C O N \epsilon}$ and show the adaptations that are needed to generalize existing theory and algorithms from $I_{A D} / I_{C O N}$ to $I_{A D \epsilon} / I_{C O N \epsilon}$. Nevertheless, both of these cases have an inherent discrepancy - the assump- 


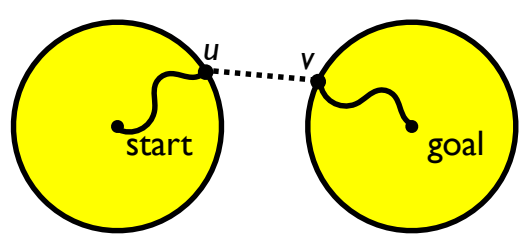

Figure 1: A path from start to goal that goes via $u$ and $v$

tions on the knowledge available to the algorithm and the assumptions on the problem instances it will run on are different. This is the main motivation for the next case.

Case 3: The algorithm is allowed to exploit the fact that both the forward and backward heuristics $\left(h_{F}\right.$ and $\left.h_{B}\right)$ are consistent. We label this case $I_{C O N} / I_{C O N}$. In this case algorithms can assume that they are running on instances from $I_{C O N}$ and we show that they can exploit a front-to-front heuristic that is induced by the consistency of the underlying heuristics.

\subsection{Necessary Expansions in Bidirectional Search}

Given an admissible heuristic, any admissible unidirectional algorithm must expand all states with $f(n)<C^{*}$ in order to prove a solution is optimal [Dechter and Pearl, 1985].

A new line of research was initiated by Eckerle et al. [2017] who generalized this theory to Bi-HS showing that necessary expansions are defined on pairs of states $u$ and $v$ in the forward and backward frontiers, respectively. Here we need to reason whether there can be an optimal path that goes from start to $u$ to $v$ to the goal, as depicted in Figure 1 . Three conditions are required to reason about potential paths between $u$ and $v$ :

$$
\begin{array}{ll}
\text { 1. } & f_{F}(u)<C^{*} \\
\text { 2. } & f_{B}(v)<C^{*} \\
\text { 3. } & g_{F}(u)+g_{B}(v)<C^{*}
\end{array}
$$

If all conditions are met, the algorithm must explore to see if there is a shorter path between $u$ and $v$. In bidirectional search, a pair of states $(u, v)$ is called a must-expand pair if all three conditions are met. Unlike unidirectional search, in a must-expand pair only one of $u$ or $v$ must be expanded, not both. An algorithm that does not expand either $u$ or $v$ cannot be admissible because it may not find an optimal solution.

\subsection{The Must-Expand Graph $\left(G_{\mathrm{MX}}\right)$}

The unique property of the must-expand condition is that it contains an or between the nodes, also a feature of the vertex cover problem. Thus, the conditions for necessary expansions can be represented as the problem of finding a vertex cover on the must-expand graph denoted by $G_{M X}$ [Chen et al., 2017].

Definition 1. The Must-Expand Graph $G_{\mathrm{MX}}$ on a problem instance is an undirected, unweighed bipartite graph. For each state $u \in G$, there are two vertices in $G_{\mathrm{MX}}$, the left vertex $u_{F}$ and the right vertex $u_{B}$. For each pair of states $u, v \in G$, there is an edge in $G_{\mathrm{MX}}$ between $u_{F}$ and $v_{B}$ if and only if $(u, v)$ is a must-expand pair.

It follows that the minimum number of node expansions required to solve a problem using $\mathrm{Bi}-\mathrm{HS}$ is determined by (a) Problem Instance

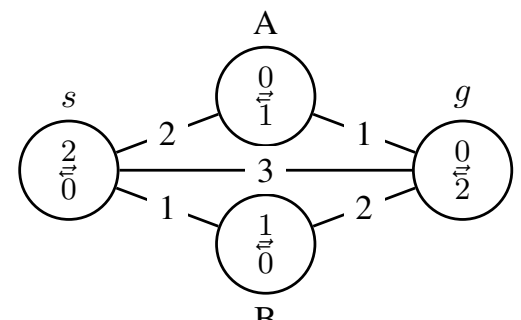

(c) $G_{\mathrm{MX} \epsilon}$

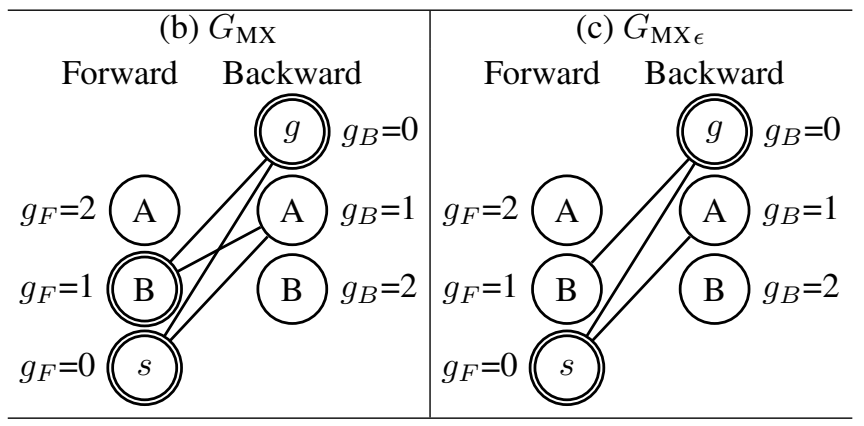

Figure 2: Case Study - Different versions of $G_{\mathrm{MX}}$

the size of the minimum vertex cover of $G_{\mathrm{MX}}$ (denoted hereafter as MVC). Naturally, the exact set of vertices in $G_{\mathrm{MX}}$ depends on the heuristic used in the problem instance. Therefore, MVC is heuristic dependent as well.

Figure 2(b) shows the $G_{\mathrm{MX}}$ graph for the problem instance in Figure 2(a) where the numbers inside nodes are the $h$ values. The left vertices are sorted by increasing $g_{F}$-costs, while the right vertices are sorted by decreasing $g_{B}$-cost. In this ordering, each horizontal pair of states $u$ and $v$ has $g_{F}(u)+g_{B}(v)=C^{*} . C^{*}=3$ in Figure 2.

\section{MVC of $G_{\mathrm{MX}}$ in the $I_{A D} / I_{C O N}$ Case}

The MVC of $G_{\mathrm{MX}}$ in the $I_{A D} / I_{C O N}$ case (Case 1) has the following properties [Shaham et al., 2017]. First, the MVC is contiguous in each direction. That is, if a node $n$ with $g_{F}(n)=k$ is in the MVC, then all nodes with $g_{F}(n) \leq k$ in $G_{\mathrm{MX}}$ must also be in the MVC, and similarly for $g_{B}$. Second, there exist thresholds $t_{F}^{*}, t_{B}^{*}$ such that (1) $t_{F}^{*}+t_{B}^{*}=C^{*}$ and (2) a forward node $u$ from $G_{\mathrm{MX}}$ is included in the MVC iff $g_{F}(u)<t_{F}^{*}$, and a backward node $v$ from $G_{\mathrm{MX}}$ is included in the MVC iff $g_{B}(v)<t_{B}^{*}=C^{*}-t_{F}^{*}$. In Section 3 we will generalize this to Case $2\left(I_{A D \epsilon} / I_{C O N \epsilon}\right)$.

To demonstrate this, consider all pairs of values of $t_{F}$ and $t_{B}$ where $t_{F}+t_{B}=C^{*}$. There is a family of contiguous vertex covers (VCs) for all such pairs $\left(t_{F}, t_{B}\right)$, where all nodes with $g_{F}<t_{F}$ in $G_{\mathrm{MX}}$ are included in the forward direction of this VC, and all nodes with $g_{B}<t_{B}$ are included in the backward direction of this VC. One such $\left(t_{F}, t_{B}\right)$ partition is the MVC and is denoted by $\left(t_{F}^{*}, t_{B}^{*}\right)$. The number of nodes of the $\left(t_{F}, t_{B}\right)$ VC partitions is determined by summing up the nodes with $g_{F}<t_{F}$ and with $g_{B}<t_{B}$. In Figure 2 , nodes that correspond to each $\left(t_{F}, t_{B}\right)$ pair are aligned horizontally. Nodes that are included in MVC have a double outline these are nodes with $g_{F}<t_{F}^{*}=2$ and with $g_{B}<t_{B}^{*}=1$. 


\subsection{Fractional MM}

$\mathrm{MM}$ is a $\mathrm{Bi}-\mathrm{HS}$ algorithm that is guaranteed to meet in the middle [Holte et al., 2017]. That is, MM will never expand a state whose $g$-value exceeds $C^{*} / 2$. Fractional MM (fMM) generalizes MM and can meet at any fraction of the optimal solution cost [Shaham et al., 2017]. $\operatorname{fMM}(p)$ uses the following priority functions on nodes in the open lists, where $0<p<1$ :

$$
\begin{aligned}
& p r_{F}(u)=\max \left(g_{F}(u)+h_{F}(u), g_{F}(u) / p\right) \\
& p r_{B}(v)=\max \left(g_{B}(v)+h_{B}(v), g_{B}(v) /(1-p)\right)
\end{aligned}
$$

$\operatorname{fMM}(p)$ expands a state with minimum priority in either direction. $\operatorname{fMM}(p)$ 's forward and backward searches meet at $\left(p \cdot C^{*},(1-p) \cdot C^{*}\right)$ by similar reasoning for why MM meets in the middle. That is, $\operatorname{fMM}(p)$ will never forward expand a state whose $g_{F}$-value exceeds $p \cdot C^{*}$ and never backward expand a state whose $g_{B}$-value exceeds $(1-p) \cdot C^{*}$. This attribute is called restrained with respect to $p$. As a result, a restrained algorithm will always return the optimal solution because it can only meet at the meeting point along the optimal path. $\mathrm{MM}$ is a special case of $\operatorname{fMM}(p)$ for $p=1 / 2$. Forward $\mathrm{A}^{*}$ and reverse $\mathrm{A}^{*}$ (searching from goal to start) are also special cases corresponding to $p=1$ and $p=0$ respectively.

For every problem instance, there exists a fraction $0 \leq$ $p^{*} \leq 1$ such that $\operatorname{fMM}\left(p^{*}\right)$ is optimally efficient and will expand the minimal number of necessary nodes - those in the MVC [Shaham et al., 2017]. In practice, however, the exact value for $p^{*}$ is not known a priori, as it depends on $C^{*}$ and $G_{M X}$. Both are not known in advance before the execution of the algorithm. But, given $C^{*}$ and $G_{M X}$ it is possible to find $p^{*}$ in time linear in the number of distinct $g$-values. Finding $p^{*}$ and then running $\mathrm{fMM}\left(p^{*}\right)$ can serve for research purposes as an oracle-any algorithm can now be compared to the theoretical optimum.

The definition of $\mathrm{EMM}$ through fractions resulted from the attempt to generalize existing algorithms such as MM and $\mathrm{A}^{*}$. Since the MVC theory uses thresholds it is more natural to use an algorithm that uses such thresholds.

\subsection{Meet at the Threshold}

Given a threshold $t$, the meet at the threshold algorithm (MT) [Shaham et al., 2018] is restrained with respect to $t$, or equivalently, MT meets at $\left(t, C^{*}-t\right)$. That is, MT will never forward expand a state whose $g_{F}$-value exceeds $t$ and never backward expand a state whose $g_{B}$-value exceeds $C^{*}-t$. $\mathrm{MT}(t)$ uses the following priority functions:

$$
\begin{aligned}
& p r_{F}(u)= \begin{cases}g_{F}(u)+h_{F}(u) & \text { if } g_{F}(u)<t \\
\infty & \text { if } g_{F}(u) \geq t\end{cases} \\
& p r_{B}(v)=\max \left(g_{B}(v)+h_{B}(v), g_{B}(v)+t\right)
\end{aligned}
$$

The same line of proof for MM and $\mathrm{fMM}$ fits here as well. The fact that MT is restrained ensures an optimal solution.

For $t=0, \operatorname{MT}(t)$ is identical to backward A*. Similarly, for $t \geq C^{*}, \operatorname{MT}(t)$ is identical to forward $\mathrm{A}^{*}$. It is easy to see that $\operatorname{MT}(t)$ will expand the same set of nodes in $G_{\mathrm{MX}}$ (not necessarily in the same order) as $\operatorname{fMM}(p)$ when $t=p \cdot C^{*}$. For example, MM is identical to $\operatorname{MT}(t)$ for $t=C^{*} / 2$. Therefore, for any value of $t, \mathrm{MT}$ will return the optimal solution. Moreover, when choosing the optimal value for $t\left(t^{*}=p^{*} \cdot C^{*}\right)$,
$\operatorname{MT}(t)$ is also optimally efficient, i.e., it expands exactly the nodes in the MVC. In the next sections we study $G_{\mathrm{MX}}$ and its MVC, and generalize MT (and $\mathrm{fMM}$, where possible) to the cases where the algorithm can assume more knowledge in the graph and on $h$.

\subsection{Other Related Bi-HS Algorithms}

Both $\mathrm{fMM}$ and $\mathrm{MT}(t)$ need a parameter as input to determine the meeting point. Another related parametric algorithm is GBFHS [Barley et al., 2018], which iteratively increases the depth of the search. It is parametric in a pre-defined split function that determines how deep to search on each side at each iteration. GBF HS with an optimal split function also converges to an MVC of $G_{\mathrm{MX}}$. However, here too, the optimal split function is not known a priori because it depends on the structure of $G_{\mathrm{MX}}$ and on $C^{*}$; both are not known in advance.

NBS [Chen et al., 2017] is a Bi-HS algorithm that aims to find some VC of $G_{\mathrm{MX}}$ by picking a must-expand pair and expanding both its vertices. Similarly, DVCBS [Shperberg et $a l ., 2019 \mathrm{~b}$ ] is a Bi-HS algorithm that grows $G_{\mathrm{MX}}$ on the fly and always expands the MVC of the growing $G_{\mathrm{MX}}$. NBS has a guarantee that the size of its $\mathrm{VC}$ is at most twice the size of the MVC. DVCBS does not have that guarantee but was reported to have a better average case performance.

A possible anomaly (first described by Holte et al. [2017]) may arise with many of these algorithms (e.g., fMM) - when using a stronger heuristic the search effort may increase. By contrast, GBFHS was shown to be inherently well-behaved (=anomaly-free). Later, Shperberg et al. [2019a] provided an important study of this anomaly and introduced improved heuristic propagation method that causes many of the algorithms (including the MM family) to be well behaved too.

\section{Case 2: Knowing $\epsilon\left(I_{A D \epsilon} / I_{C O N \epsilon}\right)$}

In many cases, the problem instance is coupled with a lower bound $(\epsilon)$ on the edge costs. For example, in unit edge-cost domains $\epsilon=1$. In other domains, one might iterate over all actions costs and take their minimum. This bound provides an admissible front-to-front heuristic of $\epsilon$ between any two distinct nodes. We now study the case where algorithms are allowed to assume the existence of $\epsilon$ but as done in previous work can still only assume an admissible heuristic. Similarly, we assume that they are evaluated in instances where the heuristic is consistent. In our notation, this case is labeled by $I_{A D \epsilon} / I_{C O N \epsilon}$. For this case we adapt the conditions for must-expand pairs in domains with front-to-front heuristics as defined by Eckerle et al. [2017] with $\epsilon$ as the front-to-front heuristic. In such cases two nodes $u, v$ are a must-expand pair if the following conditions are met:

1. $f_{F}(u)<C^{*} \quad$ 2. $f_{B}(v)<C^{*}$

3. $g_{F}(u)+g_{B}(v)+\epsilon<C^{*}$

Condition 3 is more informative than the front-to-end version of the $I_{A D} / I_{C O N}$ case (Case 1) due to the addition of $\epsilon$. In Figure 1, $\epsilon$ is a lower bound on the cost of the path from $u$ to $v$. Therefore, some edges that exist in the old $G_{\text {MX }}$ no longer exist in the new $G_{\text {MX }}$. Figure 2(c) shows such a $G_{\mathrm{MX}}$ for the instance in Figure 2(a). In this instance, 
$\epsilon=1$ and thus $(B, A)$ is no longer a must-expand pair, since $g_{F}(B)+g_{B}(A)+\epsilon=1+1+1=C^{*}$.

Similar to the $I_{A D} / I_{C O N}$ case we show that there exist thresholds $t_{F}^{*}, t_{B}^{*}$ such that a forward node $u$ from $G_{\mathrm{MX}}$ is included in MVC iff $g_{F}(u)<t_{F}^{*}$, and a backwards node $v$ from $G_{\mathrm{MX}}$ is included in MVC iff $g_{B}(v)<t_{B}^{*}$. The main difference being that this time $t_{F}^{*}+t_{B}^{*}+\epsilon=C^{*}$.

We now define the following four terms:

Definition 2.

$$
\begin{aligned}
& g_{F^{\mathrm{I}}}=\max _{u \in\left(G_{\mathrm{Mx}_{F}} \cap \mathrm{MVC}\right)}\left\{g_{F}(u)\right\} \\
& g_{F^{\mathrm{O}}}=\min _{u \in\left(G_{\mathrm{Mx}_{F}} \backslash \mathrm{MVC}\right)}\left\{g_{F}(u)\right\} \\
& g_{B^{\mathrm{I}}}=\max _{v \in\left(G_{\mathrm{Mx}_{B}} \cap \mathrm{MVC}\right)}\left\{g_{B}(v)\right\} \\
& g_{B^{\mathrm{O}}}=\min _{v \in\left(G_{\mathrm{Mx}_{B}} \backslash \mathrm{MVC}\right)}\left\{g_{B}(v)\right\}
\end{aligned}
$$

Based on these terms we define the following thresholds:

$t_{F}^{*}=\frac{\max \left\{g_{F^{\mathrm{I}}}, C^{*}-\epsilon-g_{B^{\mathrm{o}}}\right\}+\min \left\{g_{F^{\mathrm{o}}}, C^{*}-\epsilon-g_{B^{\mathrm{I}}}\right\}}{2}$

$t_{B}^{*}=\frac{\max \left\{g_{B^{\mathrm{I}}}, C^{*}-\epsilon-g_{F^{\mathrm{o}}}\right\}+\min \left\{g_{B^{\mathrm{o}}}, C^{*}-\epsilon-g_{F^{\mathrm{I}}}\right\}}{2}$

Note that $t_{F}^{*}+t_{B}^{*}+\epsilon=C^{*}$. Furthermore, these thresholds indeed satisfy the following theorem.

Theorem 1. For a forward node $u, u \in \mathrm{MVC}$ iff $g_{F}(u)<$ $t_{F}^{*}$. For a backward node $v, v \in \mathrm{MVC}$ iff $g_{B}(v)<t_{B}^{*}$.

For Figure 2(c) we have $t_{F}^{*}=1$ and $t_{B}^{*}=1$ (as opposed to the $I_{A D} / I_{C O N}$ case which had $t_{F}^{*}=2$ ).

Theorem 1 implies a simple algorithm for finding MVC given that $G_{\mathrm{MX}}$ and $C^{*}$ are both known. We iterate over all possible thresholds that satisfy $t_{F}+t_{B}+\epsilon=C^{*}$ and sum up the costs of the forward nodes $u \in G_{\mathrm{MX}}$ for which $g_{F}(u)<t_{F}$ as well as the costs of the backward nodes $v \in G_{\mathrm{MX}}$ for which $g_{B}(v)<t_{B}$. This algorithm runs with complexity linear in the number of distinct $g$-values of states in $G_{M X}$. It is similar to the Calc-VC() algorithm provided by Shaham et al. [2017].

\subsection{Meet at the Threshold $\epsilon$ (MT $\epsilon)$}

The priority function of $\mathrm{MT}$ can easily be adapted to create a new algorithm $\mathrm{MT} \epsilon$ :

$$
\begin{aligned}
& \operatorname{pr}_{F}(u)= \begin{cases}g_{F}(u)+h_{F}(u) & \text { if } g_{F}(u)<t_{F} \\
\infty & \text { if } g_{F}(u) \geq t_{F}\end{cases} \\
& \operatorname{pr}_{B}(u)=\max \left(g_{B}(u)+h_{B}(u), g_{B}(u)+t_{F}+\epsilon\right)
\end{aligned}
$$

Similar to $\operatorname{MT}(t)$ in the $I_{A D} / I_{C O N}$ case, for any given problem instance $I$ in $I_{C O N \epsilon}$ there exists $t_{F}^{*}$ such that $\mathrm{MT} \epsilon(t)$ is optimally efficient-it expands the minimal number of nodes in $G_{M X}$ of $I$. The proof for this is nearly identical to the respective proof on $\mathrm{IMM}$.

\section{Case 3: Consistency $I_{C O N} / I_{C O N}$}

As explained above, the analysis of Bi-HS by [Eckerle et al., 2017] and subsequent papers [Chen et al., 2017; Shaham et al., 2017] assumed admissible algorithms. However, they all analyzed the case where such algorithms are executed on instances from $I_{C O N}$. We next investigate the case where the algorithms know that the heuristics are consistent (i.e., they know that the instances are from $\left.I_{C O N}\right)$ and they are allowed to exploit that knowledge.

Given consistent heuristics, $\left|h_{F}(u)-h_{F}(v)\right|$ is a lower bound on the distance between $u$ and $v$, so $\left|h_{F}(u)-h_{F}(v)\right|$ can be used as a front-to-front heuristic between $u$ and $v$. Similarly, $\left|h_{B}(u)-h_{B}(v)\right|$ can be used as a heuristic as well. This was called the Add method by Kaindl and Kainz [1997].

Definition 3. (Front-to-front heuristic) For each pair of nodes $(a, b)$ define the following admissible heuristic:

$$
h_{C}(a, b)=\max \left\{\left|h_{F}(a)-h_{F}(b)\right|,\left|h_{B}(a)-h_{B}(b)\right|\right\}
$$

This heuristic results from the combination of assuming both forward and backward consistency (hence $h_{C}$ ).

\subsection{Necessary Node Expansions}

Using the conditions for must-expand pairs [Eckerle et al., 2017] in problems with $h_{C}$ as a front-to-front heuristic results in the following claim: two nodes $u, v$ are a must-expand pair if the following conditions are met:

$$
\begin{array}{ll}
\text { 1. } & f_{F}(u)<C^{*} \\
\text { 2. } & f_{B}(v)<C^{*} \\
\text { 3. } & g_{F}(u)+g_{B}(v)+h_{C}(u, v)<C^{*}
\end{array}
$$

Any pair of nodes $u, v$ that satisfy these conditions is a must-expand pair and induces an edge in $G_{M X}$ between $u$ and $v$. Condition 3 is more informative than the front-to-end version due to the addition of $h_{C}$. Therefore, some edges that exist in the old $G_{M X}$ no longer exist in the new $G_{M X}$.

\subsection{Equivalence Classes}

In the $I_{A D} / I_{C O N}$ case $h_{C}(u, v)$ did not exist. To imitate this in the $I_{C O N} / I_{C O N}$ case, we partition $G_{\mathrm{MX}}$ into equivalence classes, such that any given pair of nodes in each class $(u, v)$ has $h_{C}(u, v)=0$.

Definition 4. (heuristic equivalence) Two nodes $u, v$ are heuristically equivalent (or h-equivalent) iff $h_{C}(u, v)=0$.

Directly from the definition, two nodes $u, v$ are $h$ equivalent (belong to the same $h$-equivalence class) iff $h_{F}(u)=h_{F}(v)$ and $h_{B}(u)=h_{B}(v)$.

Lemma 2. (Restrained with respect to $t_{F Q}^{*}$ ) For every $h$ equivalence class $Q$ there exist thresholds $t_{F Q}^{*}+t_{B Q}^{*}=C^{*}$ such that MVC $\cap Q$ contains exactly the forward nodes in $Q$ for which $g_{F}<t_{F Q}^{*}$. and exactly all backward nodes for which $g_{B}<t_{B Q}^{*}$.

Another way of looking at this would be to describe $t_{F Q}^{*}$ as a function of the $h$-values of $Q$ :

Corollary 3. There exists a function $b: \mathbb{R}^{+} \times \mathbb{R}^{+} \rightarrow$ $\mathbb{R}^{+}$such that a forward node $u$ is in MVC iff $g_{F}(u) \leq$ $b\left(h_{F}(u), h_{B}(u)\right)$ and a backward node $v$ is in MVC iff $g_{B}(u) \leq C^{*}-b\left(h_{F}(u), h_{B}(u)\right)$.

Below we use $b(u)$ as shorthand for $b\left(h_{F}(u), h_{B}(u)\right)$.

We can now adapt MT to work in the $I_{C O N} / I_{C O N}$ case. We define the algorithm $\mathrm{MT}_{C O N}(b)$ with the following priorities:

$$
\begin{aligned}
& \operatorname{pr}_{F}(u)= \begin{cases}g_{F}(u)+h_{F}(u) & \text { if } g_{F}(u)<b(u) \\
\infty & \text { if } g_{F}(u) \geq b(u)\end{cases} \\
& \operatorname{pr}_{B}(u)=\max \left(g_{B}(u)+h_{B}(u), g_{B}(u)+b(u)\right)
\end{aligned}
$$




\begin{tabular}{|c||c|c|c|}
\hline Case & $I_{A D} / I_{C O N}$ & $I_{A D \epsilon} / I_{C O N \epsilon}$ & $I_{C O N} / I_{C O N}$ \\
\hline \hline MVC & Restrained & $\epsilon$-Restrained & $b \in B$ \\
\hline Optimal Alg. & fMM / MT & $\mathrm{MT} \epsilon / \mathrm{fMM} \epsilon$ & $\mathrm{MT}_{C O N}$ \\
\hline \# Clusters & $|\mathbb{G}|$ & $|\mathbb{G}|$ & $|\mathbb{G}| \times|\mathbb{H}|^{2}$ \\
\hline Complexity & $O(|\mathbb{G}|)$ & $O(|\mathbb{G}|)$ & $O\left(|\mathbb{G}|^{2} \times|\mathbb{H}|^{4}\right)$ \\
\hline
\end{tabular}

Table 1: A summarizing table. $|\mathbb{G}|$ and $|\mathbb{H}|$ are the numbers of distinct $g$ - and $h$-values respectively.

\subsection{Attributes of $b$}

As discussed above, given any threshold $t_{F} \in \mathbb{R}^{+}$, MT and $\mathrm{MT} \epsilon$ (and similarly, fMM and $\mathrm{fMM} \epsilon$ ) will always return an optimal path, although they might do more work than the minimal number of necessary expansions (as achieved by $t_{F}^{*}$ ). $\mathrm{MT}_{C O N}$, however, is not guaranteed to return an optimal path for any $b: \mathbb{R}^{+} \times \mathbb{R}^{+} \rightarrow \mathbb{R}^{+}$.

Fortunately this issue can be solved by constraining $b$ to be a member of the following set of functions $B$ :

$$
\begin{aligned}
B= & \left\{b: \mathbb{R}^{+} \times \mathbb{R}^{+} \rightarrow \mathbb{R}^{+} \mid \forall h_{F}{ }^{\prime}, h_{B}{ }^{\prime}, h_{F}{ }^{\prime \prime}, h_{B}{ }^{\prime \prime} \in \mathbb{R}^{+}\right. \\
& \left|b\left(h_{F}{ }^{\prime}, h_{B}{ }^{\prime}\right)-b\left(h_{F}^{\prime \prime}, h_{B}{ }^{\prime \prime}\right)\right| \\
& \leq \max \left\{\left|h_{F}{ }^{\prime}-h_{F}{ }^{\prime \prime}\right|,\left|h_{B}{ }^{\prime}-h_{B}{ }^{\prime \prime}\right|\right\}
\end{aligned}
$$

This is a stronger version of the global 1-Lipschitz attribute on the continuity of functions. We prove two theorems:

(1:) Given a function $b \in B, \mathrm{MT}_{C O N}(b)$ will return an optimal path on any problem instance in $I_{C O N}$.

(2:) Given a problem instance in $I_{C O N}$ there exists $b^{*} \in B$ such that $\mathrm{MT}_{C O N}(b)$ will expand the minimal number of necessary expansions on that instance.

From these theorems we can deduce that $\mathrm{MT}_{C O N}$ is optimally efficient in the $I_{C O N} / I_{C O N}$ case, provided that only functions $b \in B$ are allowed as the argument. A detailed analysis and related proofs are provided in [Shaham et al., 2018].

\section{Summary and Conclusions}

We have shown the nature of MVC for $G_{\mathrm{MX}}$ for cases with different assumptions on the knowledge of the algorithm about the heuristics used and on the nature of the underlying graph. This enriches the theory on $G_{\mathrm{MX}}$ to more cases. We have also developed MT, which is equivalent to $\mathrm{AMM}$ but is simpler for our purposes.

Table 1 summarizes our findings. Each column represents a different case. The first row presents the structure of MVC. The second row presents the optimally efficient algorithm. The third row gives the number of clusters of indistinguishable nodes in $G_{\mathrm{MX}}$, i.e., clusters that are either fully contained within an MVC or not at all. The complexity row gives the complexity of the best-known algorithm to calculate MVC given that $G_{\mathrm{MX}}$ and $C^{*}$ are known. While an algorithm for the $I_{A D} / I_{C O N}$ case and the $I_{A D \epsilon} / I_{C O N \epsilon}$ case were provided, for the $I_{C O N} / I_{C O N}$ case one should resort to the known algorithm for finding a MVC in a bipartite graph [Papadimitriou and Steiglitz, 1982].

Future work can further expand this theory to more cases such as the case where we add $\epsilon$ to the $I_{C O N} / I_{C O N}$ case. In addition, an important line will be to develop such a theory to the case where we have a full front-to-front heuristic.

\section{Acknowledgments}

The work was supported by Israel Science Foundation (ISF) grant \#844/17, by BSF grant \#2017692, by NSF grant \#1815660 and by the HUJI Cyber Security Research Center.

\section{References}

[Barley et al., 2018] Michael W. Barley, Patricia J. Riddle, Carlos Linares López, Sean Dobson, and Ira Pohl. GBFHS: A generalized breadth-first heuristic search algorithm. In SoCS, pages 28-36, 2018.

[Chen et al., 2017] Jingwei Chen, Robert C. Holte, Sandra Zilles, and Nathan R. Sturtevant. Front-to-end bidirectional heuristic search with near-optimal node expansions. In Proceedings of IJCAI, 2017.

[Dechter and Pearl, 1985] Rina Dechter and Judea Pearl. Generalized best-first search strategies and the optimality of A*. J. ACM, 32(3):505-536, 1985.

[Eckerle et al., 2017] Jurgen Eckerle, Jingwei Chen, Nathan R. Sturtevant, Sandra Zilles, and Robert C. Holte. Sufficient conditions for node expansion in bidirectional heuristic search. In ICAPS, 2017.

[Hart et al., 1968] Peter E. Hart, Nils J. Nilsson, and Bertram Raphael. A formal basis for the heuristic determination of minimum cost paths. IEEE Trans. Systems Science and Cybernetics, 4(2):100-107, 1968.

[Holte et al., 2017] Robert C. Holte, Ariel Felner, Guni Sharon, Nathan R. Sturtevant, and Jingwei Chen. MM: A bidirectional search algorithm that is guaranteed to meet in the middle. Artif. Intell., 252:232-266, 2017.

[Kaindl and Kainz, 1997] Hermann Kaindl and Gerhard Kainz. Bidirectional heuristic search reconsidered. J. Artif. Intell. Res., 7:283-317, 1997.

[Papadimitriou and Steiglitz, 1982] Christos H Papadimitriou and Kenneth Steiglitz. Combinatorial optimization: algorithms and complexity. Courier Corporation, 1982.

[Shaham et al., 2017] Eshed Shaham, Ariel Felner, Jingwei Chen, and Nathan R. Sturtevant. The minimal set of states that must be expanded in a front-to-end bidirectional search. In SoCS, pages 82-90, 2017.

[Shaham et al., 2018] Eshed Shaham, Ariel Felner, Nathan R. Sturtevant, and Jeffrey S. Rosenschein. Minimizing node expansions in bidirectional search with consistent heuristics. In SOCS, pages 81-98, 2018.

[Shperberg et al., 2019a] Shahaf Shperberg, , Ariel Felner, Nathan R. Sturtevant, Solomon Eyal Shimony, and Avi Hayoun. Improving bidirectional heuristic search by bounds propagation. In SoCS, 2019.

[Shperberg et al., 2019b] Shahaf Shperberg, Avi Hayoun, Ariel Felner, Solomon Eyal Shimony, and Nathan R. Sturtevant. Enriching non-parametric bidirectional search algorithms. In $A A A I, 2019$. 